\title{
Assessment of extinguishing efficiency of hybrid system using water mist and inert gas during class $\mathrm{A}$ fires
}

\author{
Jerzy Gałaj ${ }^{1, *}$ and Tomasz Drzymała ${ }^{1}$ \\ ${ }^{1}$ The Main School of Fire Service, Faculty of Fire Safety Engineering, 52/54 Slowackiego St., 01-629 \\ Warsaw, Poland
}

\begin{abstract}
Fixed hybrid fire extinguishing system, a new technology used in fire protection all over the world in the last years, was discussed. A fourhead twin pipe system supplied with water mist and inert gas (air or nitrogen) was applied. A pile of 50 pine wood boards was used as a combustible material (class A fire). It was located in the corner of the compartment while the nozzles were mounted symmetrically in the centre (volume suppression). The extinguishing processes differing in the proportion of water mist to gas were analysed. The extinguishing time was taken as the most important parameter indicating the extinguishing efficiency. The impact of water flow on extinguishing process was discussed. The clear dependence of extinguishing time on the water/gas ratio was proven. The best performance of the hybrid system at water flow $3 \mathrm{dm}^{3} /$ min and nitrogen as inert gas was observed. The results obtained during experiments can be useful in developing new international standards e.g. NFPA.
\end{abstract}

\section{Introduction}

International consortia, looking for new solutions, incur large financial outlays for research on innovative technologies in the field of fixed fire extinguishing devices. A wide range of firefighting devices must be adjusted so that it meets all the needs of customers. The recipient has at his disposal a number of water systems (sprinklers, sprinklers and water fog) and gas systems (inergen, FM-200, CEA 410, carbon dioxide, etc.). The use of appropriate extinguishing agent and selection of the proper installation guarantees extinguishing the fire in its initial phase, which limits the losses. Each system has advantages and disadvantages in its applications [1,2]. Fixed water-based sprinkler and fog extinguishing devices definitely dominate the market $[3,4,5]$. They are now well regulated both in Poland and in the world $[6,7,8,9,10]$. Water is ideally suited for extinguishing solid fires. The biggest advantage is its availability and extinguishing efficiency [11]. The simplicity of the installation design allows for a reasonable amount, properly protected objects. However, water is not a universal extinguishing agent. Where water is ineffective, extinguishing gases are used. Their installations are more expensive and more complicated

\footnotetext{
*Corresponding author: jgalaj@sgsp.edu.pl
} 
compared to water installations. Such solutions are designed mainly to protect archives, laboratories and server rooms. An additional advantage of extinguishing gases is the lack of conductivity of eclectic current and low fire losses [12].

Hybrid extinguishing devices are the latest application in the field of fixed fire extinguishing devices. The purpose of the system is directed to small or medium-sized rooms, eg computer server rooms $[13,14,15,16]$. The combination of the inert gas and water mist's extinguishing capabilities ensures more effective extinguishing than systems based on inert gas or water mist alone. Interest in hybrid systems is constantly growing, but so far no standard has been created that could officially launch a new era of fixed firefighting equipment. However, the lack of uniform tests does not allow the recipient to compare hybrid systems with already tested extinguishing systems. The only standard for assessing hybrid fire extinguishing systems in terms of application and extinguishing performance is FM Approval Standard 5580, "Approval Standard for Hybrid (Water and Inert Gas) Fire Extinguishing Systems" [17]. The NFPA standardization committee (NFPA 770: Standard on Hybrid (Water and Inert Gas) Fire Extinguishing Systems) also conducts work. Completion of works is planned for April 2020. Currently, the market for hybrid systems has been dominated by several companies. The constructed heads in a 50/50 volume ratio use inert gas and water. Figure 12 shows the cross section of the hybrid nozzle and its actual view. An important difference is the method of delivering the extinguishing agent to the nozzle. The solution of Ansul and Victaulic is to run two independent power lines $[13,18]$. The substances mix only in the head. Siemens has used one feed line, in which inert gas additionally drives water. The comparison of these two applications is illustrated in Figure 2. The innovative solution is the Tema Sistemi system shown in Figure 1. It uses a high-pressure water mist system with inert gas. High water pressure is achieved through the use of pump systems. All the solutions discussed earlier have a low-pressure water system [15].

Taking into account the current needs in this respect, the first in Poland pioneer study of a hybrid system using inert gas powered with inert gas was carried out at the Main School of Fire Service in Warsaw [16]. Water with gas, at the appropriate pressure, was supplied to specially designed hybrid nozzles that distributed the mixture in the fire compartment. The conclusions drawn from the research will allow to determine the advantages and disadvantages of the hybrid system under study as well as some suggestions helpful for developing new global standards.
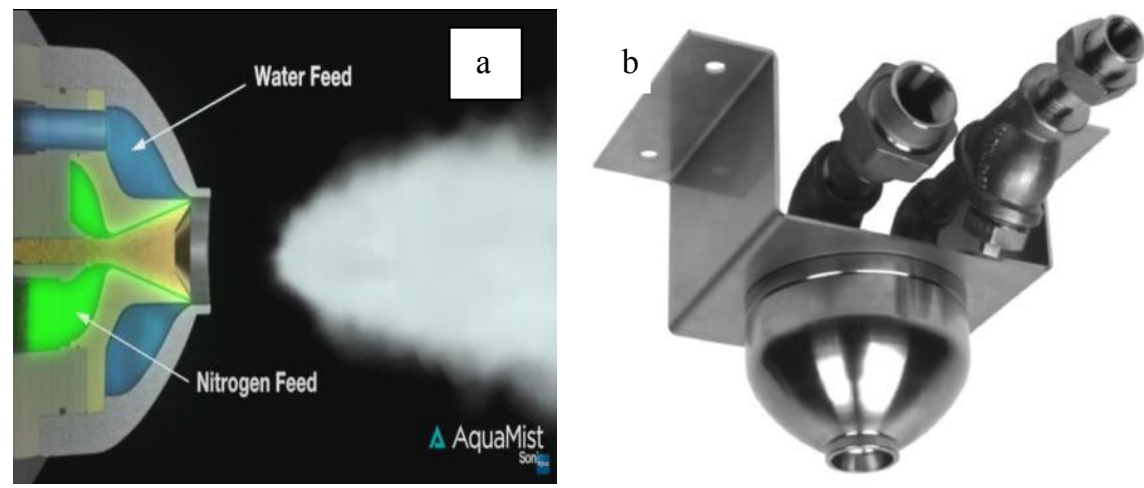

Fig. 1. Ansul's AQUASONIC system: a) nozzle cross-section, b) actual view [15]. 

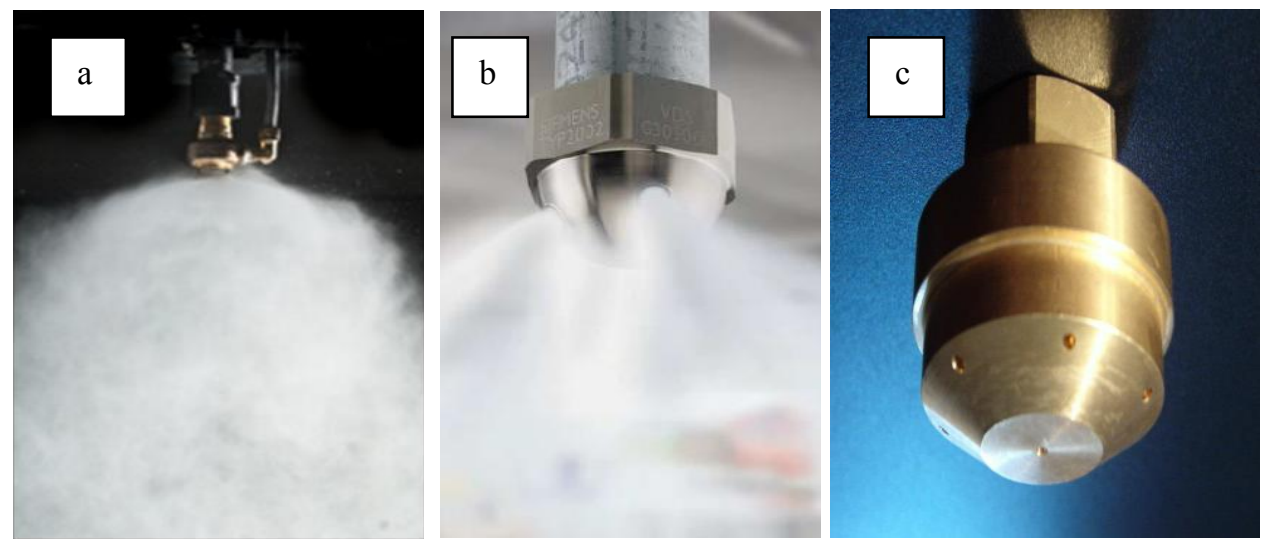

Fig. 2. Discharging of hybrid nozzles: a) Victualic Vortex system, b) Sinorix H2O Gas system from Siemens c) mist nozzles manufactured by Tema Sistemi [15].

\section{A description of experiment}

The subject of the research is a hybrid firefighting system using water fog driven by inert gas. This system operates on the basis of four hybrid nozzles 110,50 FEN-T manufactured by Telesto. Water and gas were supplied with independent supply lines in a 50/50 volume ratio. The design of the nozzles allows to bring water and gas pressure equal to $4 \pm 0.5$ bar. The technical parameters of the FEN-T 110.50 head are as follows: head weight $0.4 \mathrm{~kg}$, dimensions $70 \times 54 \mathrm{~mm}$, gas inlet diameter $1 / 4 \mathrm{inch}$, water inlet diameter $1 / 8 \mathrm{in}$, effective mist range $2.5 \mathrm{~m}$, maximum mist range $3.5 \mathrm{~m}$, droplet size from 4 to $200 \mu \mathrm{m}$, operating temperature from 10 to $700^{\circ} \mathrm{C}$, supply connections with $1 / 4 \mathrm{in}$. external thread for gas and 1/8 in. for water, fixing using M6 threaded hole from the back of the head. The tests were carried out in a closed room located in the laboratory of Technical Security Systems, which has two masonry walls lined with ceramic tiles and two glazed walls with an aluminum frame. The test chamber with dimensions of $5 \mathrm{~m} \times 5 \mathrm{~m} \times 2.5 \mathrm{~m}$ is equipped with its own mechanical ventilation system with two unloading channels. The area of ventilation ducts is $0.0324 \mathrm{~m}^{2}$ and $0.1024 \mathrm{~m}^{2}$. The water used during the tests was removed by a outflow located in the center of the floor. The doors, glazed walls and fire dampers were tight. The location of individual elements of the test stand is shown in Figure 3 (1 - power cords terminated with hybrid nozzles, 2 - test fire, 3 - carbon monoxide and oxygen detectors, 4 - thermocouple network, 5 - MDP-16 measuring module, 6 - water installation, 7 - gas cylinder system, 8 - pressure reducer, 9 - computer with software). As a combustible material, a stack consisting of 50 identical, arranged in parallel two on a special frame, pine seasonal beams measuring $30 \mathrm{~cm} \times 5 \mathrm{~cm} \times 1 \mathrm{~cm}$ and humidity of about $8 \%$ and density of about $0,55 \mathrm{~kg} / \mathrm{m}^{3}$ was used. The average weight of wooden stack was about 4,5 kg. This amount of pine wood has allowed the generation of the fire of about 100 $\mathrm{kW}$. In addition, the frame together with a pile of wood was placed on a metal tray partially filled with approximately $250 \mathrm{ml}$ of kerosene which is easily flammable. The distance of the pile stacked from the ground was $40 \mathrm{~cm}$. The tray with the pile was placed in the corner of the room adjoining two masonry walls. The distance of the test fire from the nearest hybrid nozzle was about $150 \mathrm{~cm}$. The view of the stack before the test is shown in Figure 4. 


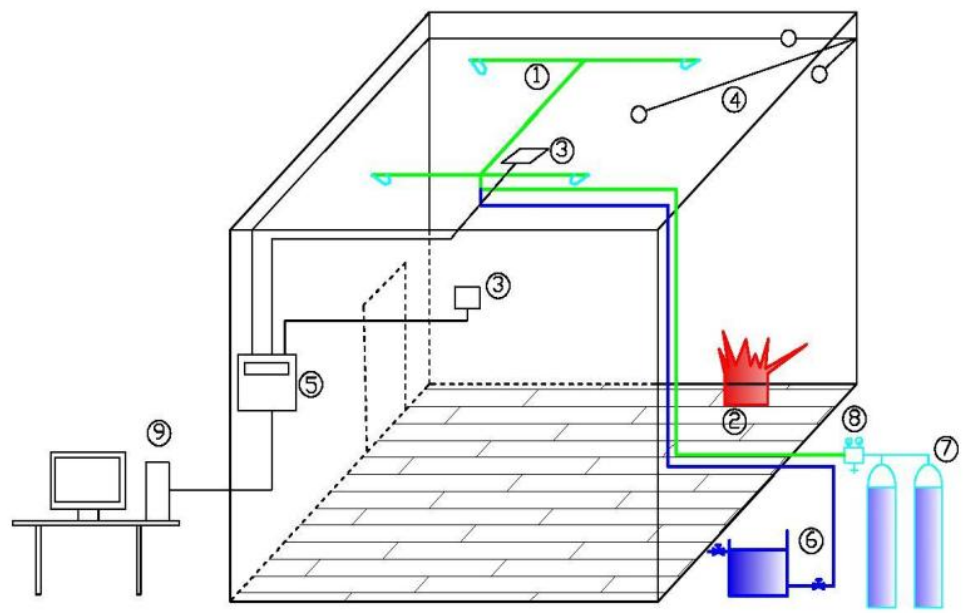

Fig. 3. Block diagram of the test stand.

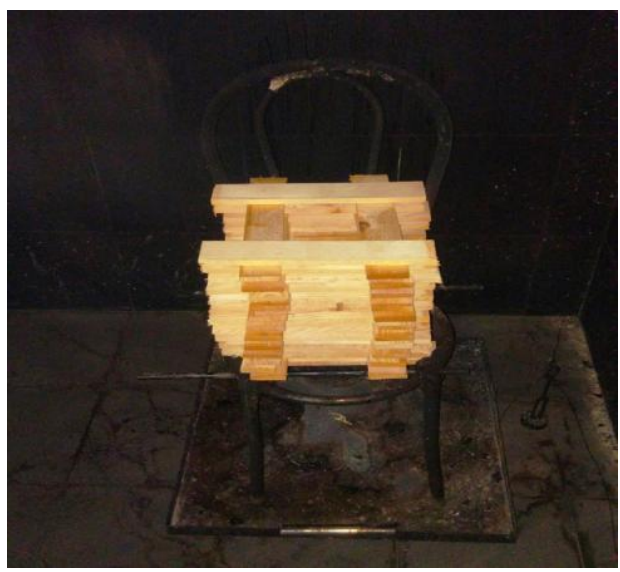

Fig. 4. A view of stack before testing.

The hybrid system under study consisted of a separate water and gas section. The entire installation was suspended on steel wires at a distance of $40 \mathrm{~cm}$ from the ceiling. The water section was powered by a pumping system located in an adjacent room. The gas section was supplied from a set of cylinders containing air or nitrogen at a pressure of 200 bar connected in parallel, located outside the laboratory. They led gas to the reducer, which at a suitable pressure of 4 bar delivered it to the hybrid nozzles. The chamber has 26 thermocouples of type TKP CZAKI $(\mathrm{NiCr}-\mathrm{NiAl})$ for temperature measurement and two electrochemical oxygen sensors of type Gazex DG-P9 E/N and one electrochemical carbon monoxide sensor Gazex DG-P2 E/N designed to measure the concentration of oxygen and carbon monoxide respectively. The parameters of the sensor DG-P9 E/N are as follows: range $0-25 \%$, accuracy $0,2 \%$, response time $30-90 \mathrm{~s}$, relative error $< \pm 10 \%$, working temperature from $-25^{\circ} \mathrm{C}$ to $50^{\circ} \mathrm{C}$ and tightness class IP44. The location of the thermocouples is shown in Figure 5. Each of them has been assigned a number corresponding to the appropriately programmed number in the Advantech ADAMViev program, which was used to record and process the measured data. The measurement was started when the wood pile was set on fire and ended when it was extinguished. Extinguishing (simultaneous application of water and gas to the system) was started when any thermocouple reached $200^{\circ} \mathrm{C}$. The program recorded temperature measurements every 
1 second, saving each sample in a separate file. The extinguishing time was also recorded by the computer with the same accuracy.
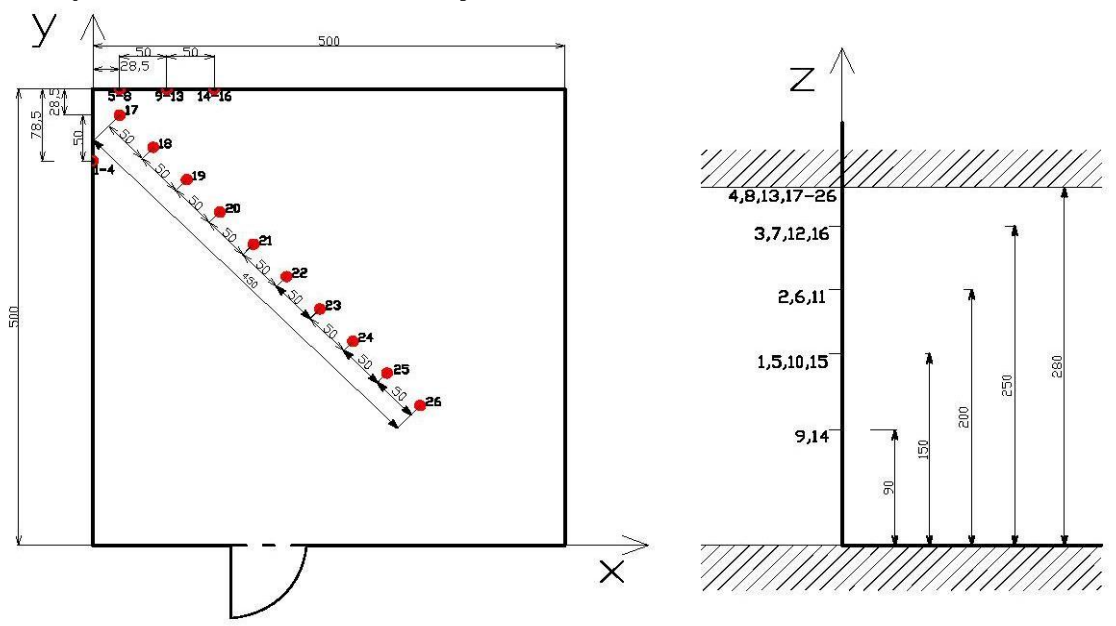

Fig. 5. Diagram of thermocouples arrangement in the horizontal and vertical plane.

\section{Results of experiment}

The extinguishing time is one of the most important parameters illustrating the extinguishing effectiveness of the tested installation. Installations differ from each other in terms of construction and firefighting medium, but relatively in general looking at fire situations, the most important issue is to reduce or extinguish a fire as soon as possible. A total of 37 tests of the nitrogen-driven hybrid system were carried out at various water mist performances. For comparison, several tests of air-driven water fog were carried out. In order to systematize the results, a few characteristic times were distinguished, such as: start marking the start of the extinguishing procedure, stop marking the end of the extinguishing procedure and the extinguishing time being the difference between the end and the beginning of extinguishing. From the tests conducted for the same gas and water mist efficiency, the one selected was the one for which the extinguishing time was closest to the average extinguishing time calculated for all tests. The extinguishing times obtained and the time moments corresponding to the beginning and end of extinguishing for tests with only nitrogen without water and with nitrogen, in which four different water flows were used (3 $\mathrm{dm}^{3} / \mathrm{min}, 1.5 \mathrm{dm}^{3} / \mathrm{min}, 1 \mathrm{dm}^{3} / \mathrm{min}$ and $0.5 \mathrm{dm}^{3} / \mathrm{min}$ ) and air, during which two different water flows were applied $\left(3 \mathrm{dm}^{3} / \mathrm{min}\right.$ and $\left.1.5 \mathrm{dm}^{3} / \mathrm{min}\right)$ are collected in Table 1 . In addition, Figure 6 shows the extinguishing times corresponding to these tests. Despite the fact that attempts were made to provide comparable conditions for the development of a fire (the same amount of the same type of pine wood arranged in the same way, the same amount of liquid flammable, the same localization of combustible material) in one case a significant difference was achieved between the times of reaching the limit temperature in the same point of the room (about $180 \mathrm{~s}$ ). It could have been influenced by factors (e.g. local airflow) that determine that a fire in the same room with the same equipment can evolve at different rates each time and it is practically impossible to repeat it. However, the aim of this work was not to analyze the fire development, but to extinguish it. Hence the most important changes were in the temperature and other parameters after starting the extinguishing process. To analyze the effect of the oxygen concentration level on the extinguishing process, Figure 7 shows the charts illustrating average oxygen concentration when using only nitrogen as well as water fog driven by nitrogen or air. 
Table 1. Characteristic time parameters obtained during tests.

\begin{tabular}{|c|c|c|c|c|c|c|}
\hline \multirow[b]{2}{*}{$\begin{array}{c}\text { Water } \\
\text { flow } \\
{\left[\mathrm{dm}^{3} / \mathrm{min}\right]}\end{array}$} & \multicolumn{3}{|c|}{ Nitrogen } & \multicolumn{3}{|c|}{ Air } \\
\hline & $\begin{array}{c}\text { start } \\
{[s]}\end{array}$ & $\begin{array}{c}\text { stop } \\
{[\mathrm{s}]}\end{array}$ & $\begin{array}{l}\text { extinguishing } \\
\text { time } \\
{[s]}\end{array}$ & $\begin{array}{c}\text { start } \\
{[\mathbf{s}]}\end{array}$ & $\begin{array}{c}\text { stop } \\
{[\mathrm{s}]}\end{array}$ & $\begin{array}{c}\text { extinguishing time } \\
{[\mathrm{s}]}\end{array}$ \\
\hline 3.0 & 265 & 470 & 205 & 276 & 500 & 224 \\
\hline 1.5 & 351 & 566 & 215 & 169 & 608 & 439 \\
\hline 1.0 & 320 & 611 & 291 & - & - & - \\
\hline 0.5 & 328 & 634 & 306 & - & - & - \\
\hline 0.0 & 271 & 620 & 349 & - & - & - \\
\hline
\end{tabular}

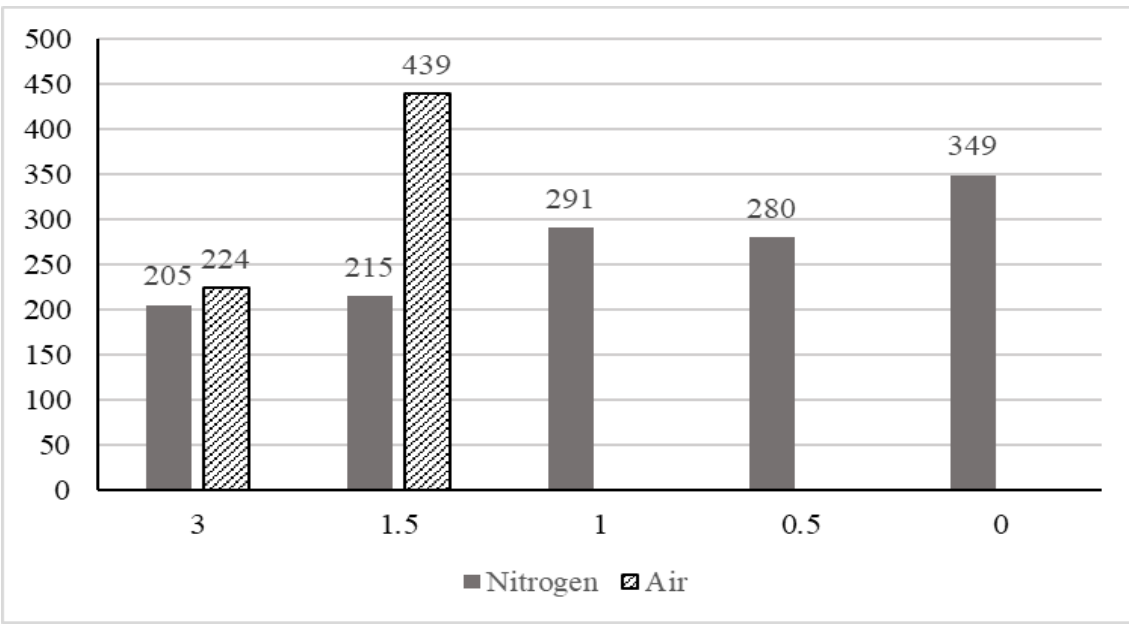

Fig. 6. Extinguishing times for a hybrid system using only nitrogen and water mist driven by nitrogen for four different water flows and driven by air for two different water flows.

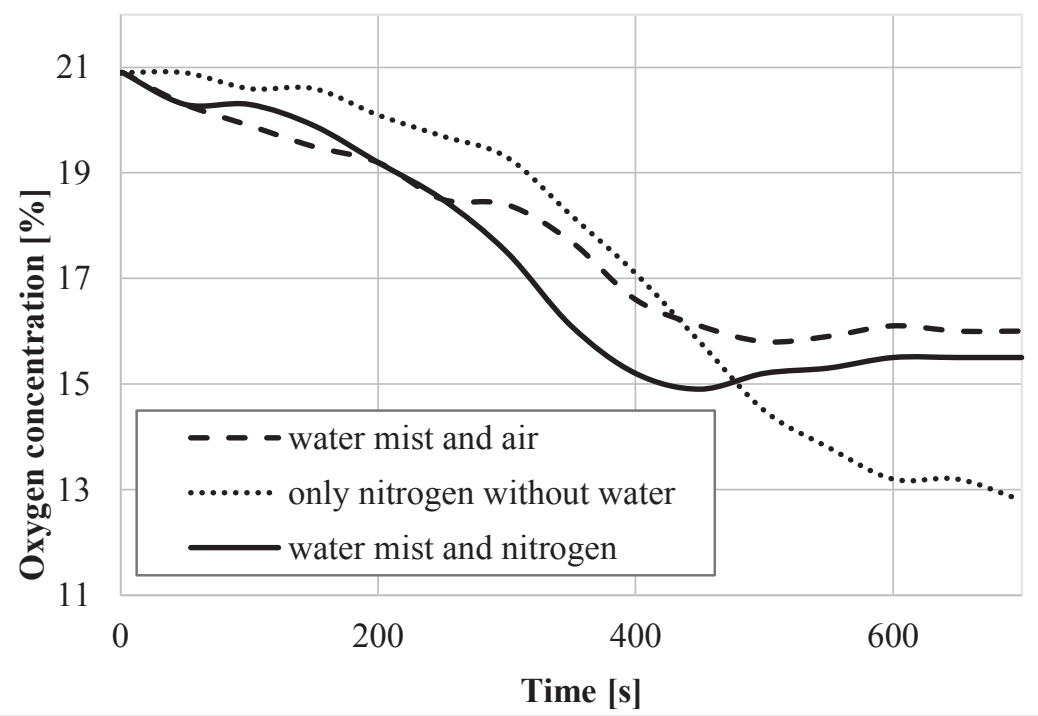

Fig. 7. Average oxygen concentrations during extinguishing by hybrid system using nitrogen without water and nitrogen-driven water fog or air-driven water fog. 


\section{Conclusions}

When searching for the best solutions for fixed fire extinguishing systems, the most important factors are their fire extinguishing efficiency and price. If you want to properly protect an object or room, you often have to pay big costs for installation and extinguishing agent. The hybrid fire suppression system that uses water mist and nitrogen, introduced to the market, is a novelty among fire protection systems. However, we must answer the question: is the hybrid system really better than the already known fire protection and is it worth investing in it? The tests of this system, carried out for the first time in Poland, prove its effectiveness. On the basis of the results obtained and in particular the analysis of firefighting times using a hybrid system, in which two types of gas were used (nitrogen and air) and several different water mist flows ranging from $0.5 \mathrm{dm}^{3} / \mathrm{min}$ to $3 \mathrm{dm}^{3} / \mathrm{min}$, the following conclusions were made:

1. A hybrid system using nitrogen-driven water fog has a higher extinguishing efficiency than a system using air-driven water fog or using only nitrogen without water. The difference between the average time of extinguishing using nitrogen and air at a flow of $3 \mathrm{dm}^{3} / \mathrm{min}$ was about $20 \mathrm{~s}$, and at a flow of $1.5 \mathrm{dm}^{3} /$ min already over $220 \mathrm{~s}$. The longest average extinguishing time was obtained when using only nitrogen without water mist. It was longer than about $415 \mathrm{~s}$ from the shortest extinguishing time using nitrogendriven water fog and about $396 \mathrm{~s}$ from the shortest extinguishing time with air-driven water fog.

2. As expected, the shortest average extinguishing time equal to $205 \mathrm{~s}$ was obtained for water flow equal to $3 \mathrm{dm}^{3} /$ min driven by nitrogen. In the case of air-driven for the same water flow, a fire-fighting time longer about 20 seconds was obtained. Comparing the extinguishing times at different water flows, it can be concluded that with its decrease, the extinguishing time increases. For the lower flow of water driven by nitrogen, the times were longer about: $10 \mathrm{~s}$ for $1.5 \mathrm{dm}^{3} / \mathrm{min}, 86 \mathrm{~s}$ for $1 \mathrm{dm}^{3} / \mathrm{min}$ and $101 \mathrm{~s}$ for $0.5 \mathrm{dm}^{3} / \mathrm{min}$ respectively. From this it results that when water flow is less than or equal to $1 \mathrm{dm}^{3} / \mathrm{min}$, extinguishing is much less effective than at higher flows.

3. Nitrogen consumption during extinguishing at water flow $3 \mathrm{dm}^{3} /$ min was about 3 times lower than extinguishing with nitrogen alone.

4. During fire suppression with air-driven water mist, higher concentrations of oxygen in the room were observed (the lowest was about 16\%), compared to a nitrogen-driven water-mist system (the lowest was about 15.5\%) and nitrogen alone the lowest concentration of about $13 \%$ was obtained (close to the concentration limit at which the fire goes out spontaneously due to insufficient oxygen). As a result of research conducted as part of the FM Global certification, the following ranges of oxygen concentrations were obtained: in the system of water fog measured by the "dry" method, from $14.9 \%$ to $16.2 \%$ and "wet" from $12.4 \%$ to $12.6 \%$, in the gas system from $12.3 \%$ to $12.6 \%$ and from $12 \%$ to $12.1 \%$, in the hybrid system from $13.5 \%$ to $14.6 \%$ and from $12.2 \%$ to $12.3 \%$ respectively.

\section{References}

1. E. Arthur, Fire Protection Handbook Nineteenth Edition (Cote Quincy, Massachusetts, 2003)

2. T. Sowa, Fire Safety Techn. 17, 1 (2010)

3. P. Zbrożek, J. Prasuła, Fire Safety Techn. 15, 3 (2009)

4. W. Wnęk, P. Kubica, M. Basiak, Fire Safety Techn. 27, 3 (2012) 
5. U. Schremmer, Water mist technique - possibilities and limits of use, [in:] Proceedings of IV Conference Fixed Fire-fighting equipments, Józefów (2004)

6. NFPA 13:2010

7. VdS CEA 4001pl: Guidelines for sprinkler system. Designing and (2010)

8. PN-EN 12845+A2:2010

9. NFPA 750:2010

10. NFPA 2010, Standard for Fixed Aerosol Fire-Extinguishing Systems

11. J. Gałaj, T. Drzymała, R. Šukys, P. Tofiło, J. Civil Engin. Management 24, 1 (2018)

12. I. Schlosser, Standards and guidelines for gas extinguishing devices, [in:] Proceedings of IV Conference Fixed Fire-fighting equipments, Józefów (2004)

13. P. Raia, M. Gollner, Literature Review on Hybrid Fire Suppression Systems, (University of Maryland, Fire Protection Research Foundation, College Park (MD) 2014)

14. R. Ballard, Introduction to Hybrid Extinguishing Technologies, MEMORANDUM to Technical Committee on Hybrid Extinguishing Systems, part 5b. St. Louis (MO) (2015)

15. P. Wolny, N. Tuśnio, Research and development of extinguishing hybrid systems, Proceedings of Conference Development tendencies in fire-fighting, Cracow (2017)

16. T. Drzymała, J. Gałaj, Chem. Indust. 97, 6 (2018)

17. FM Approvals 5580, Approval Standard for Hybrid (Water and Inert Gas) Fire Extinguishing Systems, Factory Mutual Global, Johnston (RI) (2012)

18. R. Ballard, Victaulic Vortex (C) Hybrid Fire Extinguishing Technology and NFPA 770 Standard Development, Great Plains Fire Suppression Symposium, Omaha (NE) (2016) 